\title{
Robot-assisted humanized passive rehabilitation training based on online assessment and regulation
}

\author{
Lizheng Pan ${ }^{\mathrm{a}, \mathrm{b}, *}$, Aiguo Song ${ }^{\mathrm{b}}$, Suolin Duan ${ }^{\mathrm{a}}$ and Baoguo $\mathrm{Xu}^{\mathrm{b}}$ \\ ${ }^{a}$ School of Mechanical Engineering, Changzhou University, Changzhou 213164, P.R. China \\ ${ }^{b}$ School of Instrument Science and Engineering, Southeast University, Nanjing 210096, P.R. China
}

\begin{abstract}
Robot-assisted rehabilitation has been developed and proved effective for motion function recovery. Humanization is one of the crucial issues in the designing of robot-based rehabilitation system. However, most of the previous investigations focus on the simplex position control when comes to the control system design of robot-assisted passive training, and pay little attention to the dynamic adjustment according to the patient's performances. This paper presents a novel method to design the passive training system using a developed assessing-and-regulating section to online assess the subject's performances. The motion regulating mechanism is designed to dynamically adjust the training range and motion speed according to the actual performances, which is helpful to improve the humanization of the rehabilitation training. Moreover, position-based impedance control is adopted to achieve compliant trajectory tracking movement. Experimental results demonstrate that the proposed method presents good performances not only in motion control but also in humanization.
\end{abstract}

Keywords: Rehabilitation robot, humanized rehabilitation training, online assessment, stroke

\section{Introduction}

Stroke is a common cerebrovascular disease, and it usually causes motion dysfunction or permanent disability. Approximately 780,000 Americans suffer from a stroke each year, resulting in reduced mobility and disabilities $[1,2]$. In China, there are about 2,000,000 people suffering from a stroke each year, and approximately 66\% survivors following with deficits of motor function [3]. Motion disability results in serious inconvenience in daily life and heavy burden in psychology. Currently, how to help the people with motion disabilities recover effectively, it is a worldwide problem.

Fortunately, clinical outcomes have verified that the repetitive and intensive rehabilitation training is helpful to improve the motion functions according to the brain plasticity theory [4]. One of the aims of developing rehabilitation training robot is to assist therapists and serve the patients to regain their motion functions. Many researches have done great contributions in mechanism design, control strategies, rehabilitation methods, rehabilitation assessment, and so on [5-9]. Generally, the

\footnotetext{
* Address for correspondence: Lizheng Pan, School of Mechanical Engineering, Changzhou University, Changzhou 213164, China. Tel.: +86+15961282875; Fax:+86-519-81169810; E-mail: plz517@sina.com.cn.
}

0959-2989/15/\$35.00 @ 2015 - IOS Press and the authors. 
rehabilitation trainings are divided into passive and active recovery trainings. For active training, many research works focus on developing control strategies, such as based on vision feedback (designing training games, error augment), based on exerting assistive force (planning trigger region, or according to the performances). The developed control strategies effectively evoke patient's active participation, and make the training more intelligent and safer in some degree. Passive recovery training is usually adopted at early stage for patients without any motion ability. The aim of passive training is to reduce the muscular tension and increase impaired-limb movable region [10]. For passive training, one control system is usually designed to control the robot smoothly and stably to draw the impaired limb moving along a predefined trajectory with the designed position controller. Thus, in passive training mode, the existing investigations mainly focus on developing control strategies based on position control and planning trajectory [11, 12]. O'Malley, et al designed a PD trajectory controller with fixed gain to mange the Rice Wrist to serve the passive training [13]. Clumer, et al designed a 6-DOF iPAM rehabilitation system and adopted admittance controller to mange the passive training, the experimental results demonstrated that the admittance control method was able to serve the training stably and smoothly [14]. In general, the existing control methods for passive training are able to effectively stretch the impaired limb moving along the designed trajectory. According to the clinical therapy, humanization is one of the crucial issues in the designing of robot-based rehabilitation system. When doing training, the designed controller should adaptively adjust the training range and speed according to the online specific conditions, does as the physiotherapist. However, the existing control systems for passive training pay little attention to the point of humanization.

Thus, this paper proposes a novel method for control system design, which serves with humanized passive rehabilitation trainings based on online assessing the subject's performances. In the designed system, the developed assessing-and-regulating section online monitors and assesses the subject's performances during the robot-assisted training, and the training range and motion speed are adaptively adjusted according to the assessment results with the designed motion regulating mechanism, as what the physiotherapist does in clinical one-to-one therapy.

\section{Motion assessment}

In clinical therapy, the actual physical state of training impaired-limb (PSTIL) is dynamically changed due to the uncertain internal or external disturbances [11]. Moreover, the planned training range and speed is usually adjusted in real time according to the subject's state of the illness and training performances. Thus, the motion assessment plays an important role on achieving robotassisted humanized training. In this research, the motion assessment includes two parts of work (see Figure 1), namely assessment of the PSTIL which works for online regulating the training speed, and assessment of the motion performances which works for adjusting the training range.

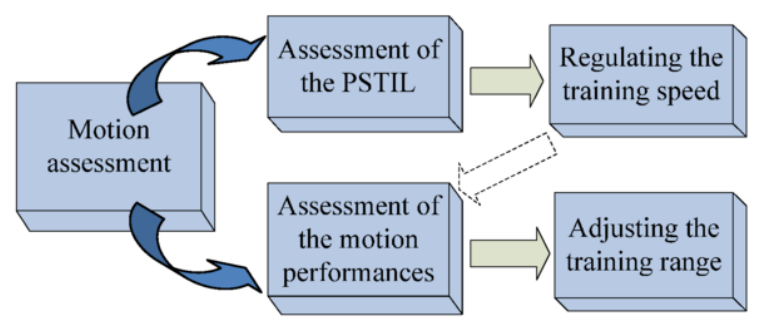

Fig. 1. Motion assessment. 


\subsection{PSTIL}

Robot-assisted rehabilitation training is a typically dynamic human-machine interaction process. Thus, in a sense, the uncertain internal or external disturbances affect the control performances of the motion training [11], which is usually reflected on the position-velocity tracking performances during the passive training. In our previous work [15], we adopted the position-velocity tracking features to assess the PSTIL, which is combined with the fuzzy logic reasoning. Subsection sliding mean square error (SMSE) is employed to extract the features, which is expressed as

$$
\left\{\begin{array}{l}
\delta_{k}=\sqrt{\frac{\sum_{i=1}^{n}\left(\bar{x}_{k}-x_{k-i}\right)^{2}}{n-1}} \\
\bar{x}_{k}=\frac{1}{n} \sum_{i=1}^{n} x_{k-i}
\end{array}\right.
$$

where, $n$ is the length of the subsection window, $x_{k-i}$ is the value of $(k-i)^{t h}$ sample data, $\bar{x}_{k}$ is the mean of $k^{\text {th }}$ subsection, and the $\delta_{k}$ is the corresponding feature of the $k^{\text {th }}$ sample data.

And fuzzy logic reasoning with double inputs and single output type is employed to evaluate the PSTIL, in which the motion features of position and velocity tracking errors are used as the two inputs, and the corresponding output reflects the PSTIL during the training in real time.

In clinical therapy, the physiotherapist usually online adjusts the training speed according to the PSTIL during the traditional one-to-one training. Thus, in this paper, the online assessment of the PSTIL is adopted to regulate the robot-assisted motion parameters in real time, and achieves different degrees of reduction in speed, stopping the training, or serving in emergency mode.

\subsection{Motion performances}

The training motion performances, in a manner, synthetically reflex the state of the illness, patient's real-time conditions and the rationality of the training programs, and so on. The monitoring and assessing of the motion performances provide an important basis for determining and adjusting the training task according to the patient's specific case, which is very helpful to improve the training efficacy.

In general, there is little caused discomfort disturbance when the predefined range of training movement (ROTM) is ideally suitable for the patient. Otherwise, the discomfort disturbance is usually caused during the training when undergoing a ROTM beyond the patient's capacity. In other word, the ROTM is suitable or not, which is reflected on the motion performances. In this paper, the regulating mechanism is designed, and the ROTM is adjusted in accordance with the regulating mechanism.

\section{Control system design of passive rehabilitation training}

Currently, the existing robot-assisted passive training is usually executed with tracking the predefined trajectory using various developed motion control methods, and the predefined trajectory is changeless during the training $[11,16]$. In this research, based on clinical application, we proposed a system design method for robot-assisted humanization passive training, which online regulates the 
training motion parameters with developed real-time assessing-and-regulating section.

\subsection{Control method}

The robot-assisted rehabilitation training is applied to serve the patients with motor dysfunction which is usually caused by neurological damage. Due to the partial damage of the brain function, the central nervous system (CNS) of the subject is not stable as the one of the health. The patient is more susceptible to injury during the training, especially on the early stage, in which the state of the illness is usually serious, and the condition of the patient is unstable. Thus, the suitable compliant behavior of the physical interactive training is greatly addressed during the control system design. Impedance control [17] is a developed method to achieve the interactive compliance inherently. In this research, the position-based impedance control is employed to manage the trajectory tracking motion.

According to $[18,19]$ and our previous experimental investigation $[15,20]$, a mass-damper-spring relationship between the position and the force is established for the rehabilitation robot by impedance control, which is represented as

$$
\begin{gathered}
\Delta F=M_{d} \Delta \ddot{X}+B_{d} \Delta \dot{X}+K_{d} \Delta X \\
\Delta X=X_{d}-X, \Delta \dot{X}=\dot{X}_{d}-\dot{X}, \Delta \ddot{X}=\ddot{X}_{d}-\ddot{X}
\end{gathered}
$$

where $X_{d}, \dot{X}_{d}, \ddot{X}_{d}$ are the desired position, velocity and acceleration; $X, \dot{X}, \ddot{X}$ are the corresponding actual information of rehabilitation manipulator; $M_{d}, B_{d}, K_{d}$ are the desired matrices for inertia, damping and stiffness, respectively; $\Delta F$ is the variance in force.

The impedance controller is employed to dynamically regulate the desired position according the force, which improves the interactive compliance. The proportional-integral-derivation (PID) position controller is employed to execute the position tracking.

In this research, the assessing-and-regulating section is developed to achieve more intelligent and humanized robot-assisted passive training. According to the clinical therapy, the developed assessingand-regulating section online regulates the training motion speed according to the assessment result of the PSTIL and adjusts the ROTM on the basis of the assessment of the motion performances. The overall block diagram of the proposed control system design method with assessing-and-regulating section is shown as in Figure 2. It mainly includes four sections, namely motion assessment, motion regulation mechanism, impedance control regulator and PID controller. The motion assessment online assesses the PSTIL with fuzzy logical reasoning using the extracted position-velocity characteristics. The online motion regulating mechanism regulates the training motion speed and range, depending on the online evaluated PSTIL and motion performances of the last two sessions. The impedance control regulator and PID position controller are adopted to achieve interactive compliance and stretch the impaired limb executing the trajectory tracking.

\subsection{Regulating mechanism for training motion}

\subsubsection{Regulation of the training motion speed}

During the traditional one-to-one training by hand, the physiotherapist usually adjusts the stretching speed according to the patient's real-time conditions, in order to avoiding doing damage to the impaired limb. In this research, the aim of the regulation for the training speed is to achieve humanized passive training just as what the physiotherapist does. The PSTIL is varied during the 


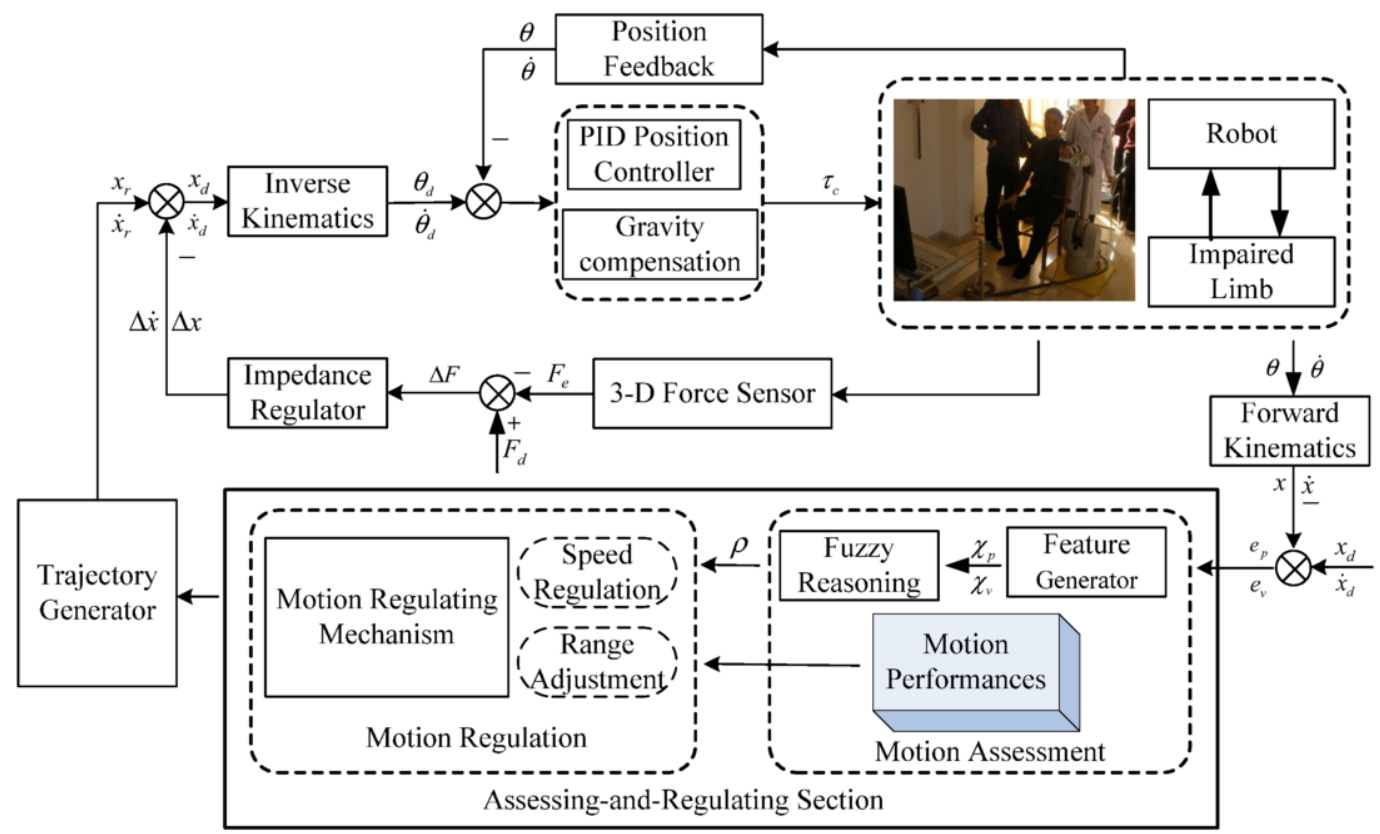

Fig. 2. Control system block diagram.

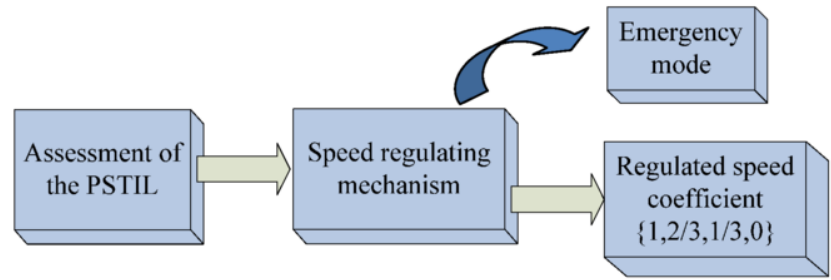

Fig. 3. Regulating motion parameter.

robot-assisted training due to the existing disturbances in different degrees. The online assessment of the PSTIL provides available ground to regulate the robot-assisted motion parameters in real time, and make the rehabilitation system serve with speed reduction in different degrees, stopping the training, or being emergency mode, which is shown as in Figure 3. Therefore, the robot-assisted training speed should be adaptively slowed down or stopped when the impaired limb is undergoing the pose-position change or forearm slight quiver, and the emergency mode should be trigged under a sudden twitch or emergency. According to the practical application in clinical therapy, the training parameter for motion speed is regulated with four grades, namely $\{1,2 / 3,1 / 3,0\}$. Under emergency, the system works in emergency mode, in which the manipulator floats and follows the impaired limb movement freely without interactive force. The online regulation of motion parameters makes the robot-assisted training more humanization, and effectively prevents the damage to the impaired limb under uncertain internal or external disturbances.

\subsubsection{Adjustment of the ROTM}

As mentioned in section 2.2, a conclusion whether the ROTM is suitable or not can be made by analyzing the motion performances. In clinical therapy, in order to achieve the best therapeutic effect, the ROTM should be adjusted to match with the patient's conditions. In this research, to achieve more 
Table 1

Regulation rules of decreasing ROTM

\begin{tabular}{|c|c|c|c|c|c|c|c|c|c|}
\hline Number of planning points & 268 & $\rightarrow$ & 203 & $\rightarrow$ & 136 & $\rightarrow$ & $170(0.8 \mathrm{v})$ & $\rightarrow$ & $156(0.8 \mathrm{v})$ \\
\hline ROTM & {$[-5.37,-3.8]$} & & {$[-5.3,-3.9]$} & & {$[-5.2,-4.0]$} & & {$[-5.2,-4.0]$} & & {$[-5.1,-4.1]$} \\
\hline
\end{tabular}

intelligent and humanized motion adjustment of training rage, not only the ROTM but also the influence of the global predefined motion speed is considered during the design of the regulating mechanism for ROTM. Combining the clinical application, the specific rules of assessing the motion performances and adjusting the ROTM are described as following.

Rules for assessing and adjusting:

(1) During one training session, when the discomfort disturbances cause the training in pausing more than 3 times, it indicates that the predefined ROTM is beyond the patient's motion capacity. Thus, the ROTM should be turned down in current and next training sessions.

(2)In the regulating mechanism, the regulation of ROTM plays a main role, while taking into account the global speed planning. When the ROTM is small, ROTM less than [-5.2, -4.0] rad in vertical training, it usually means that the state of the illness is more serious, and the training should be predefined at slow speed (decreasing the global predefined speed). The regulation rules in decreasing ROTM for vertical training are as in Table 1.

(3)When the training is served without any reducing the speed, pausing, and so on, in two consecutive training sessions, it indicates that the subject does great well with current ROTM. The ROTM should be increased in the next session, and the regulation for increasing ROTM is implemented in the opposite direction to (2).

(4)The ROTM is without adjustment except under the conditions (1) and (3).

\section{Experiments and results}

\subsection{Experiment system}

In our previous work [15], we have constructed the WAM upper-limb rehabilitation system as shown in Figure 4 which mainly consists of an external PC, the Barrett WAM, a self-developed 3-D force sensor, and arm support device. The Barrett WAM can work in a wide 3-D space with four degree-of-freedom (DOF) rotating joins, at the same time with good performance in back drivability. During the training, the four driver motor angles can be measured to detect the position of each joint in real time, and the control torque can be set to provide the joint control. Meanwhile, the interactive force between the impaired limb and the WAM end-effector is recorded by the self-developed 3-D force sensor. The arm support device well supports the forearm of the subject to execute the robotassisted passive training. More information about the WAM rehabilitation system was introduced in $[15,20]$.

In terms of the rehabilitation system software, the proposed control strategy is developed on the external PC which runs with Ubuntu Linux system. A graphical user interface (GUI) is developed to visually display the related functions, such as setting the trajectory type, training model, gravity compensation coefficient, etc. During the software design, the real-time module Xenomai and multithread method are adopted to manage all the system tasks, which is helpful to realize the real-time 


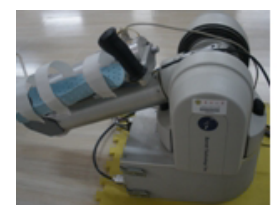

Barrett WAM

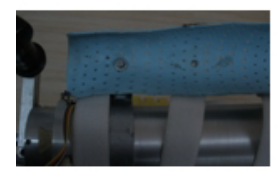

Arm Support

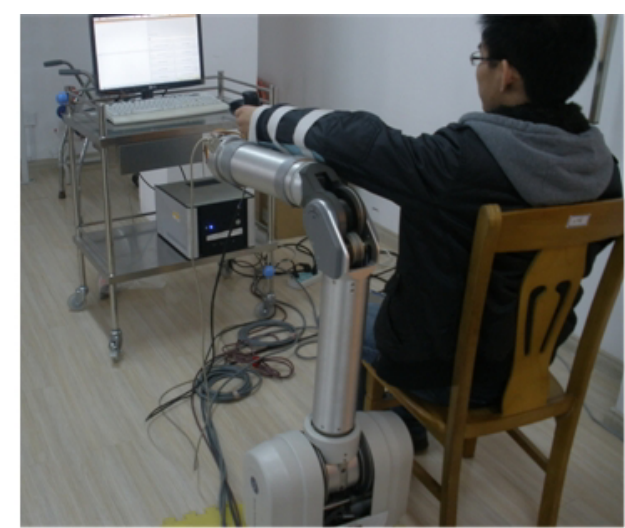

Fig. 4. WAM upper-limb rehabilitation system.

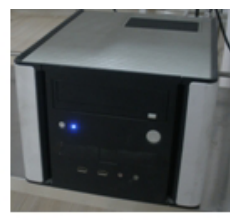

PC

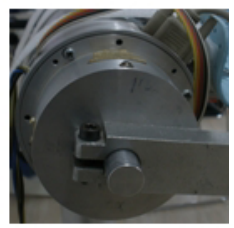

3-D Force Sensor

control.

\subsection{Experiment scheme}

In this research, to verify the effectiveness of the proposed control system design method combining with a developed online assessing-and-adjusting section, the training trajectory of elbow extension/flexion in vertical was predefined. The single session time, planning points, and the movable range expressed in the WAM Arm's world for the predefined training trajectory were $14.3 \mathrm{~s}, 286$, [$5.37,-3.8]$ rad, respectively.

The quantitative and qualitative tests were schemed and carried out to investigate the control performances in stability and humanization. A healthy adult male volunteer was inducted to undergo the experiments to present and analyze the control performances. In quantitative experiment, the $10 \mathrm{~N}$ disturbance with one second was employed to present the control stability, which was exerted in the opposite direction of the movement. In qualitative experiment, the subject was inducted to deliberately simulate the different PSTIL (without disturbance, small disturbance, and large disturbance) during the training, to demonstrate and analyze the stability and humanized regulation of the rehabilitation control system.

\subsection{Results}

The quantitative and qualitative experiments were carried out to investigate the control performances of the proposed system design method. In terms of quantitative experiments, the control performance under one-second $10 \mathrm{~N}$ disturbance was analyzed and compared between the traditional position-based impedance control and the proposed method combining with an online assessing-andadjusting section. In the vertical elbow extension/flexion training, the tracking performances presenting with respect to the fourth joint of the WAM were studied. The experimental results are shown in Figure 5. Furthermore, the maximum of the absolute error (MAE), the sum of absolute error (SAE) and the sum of absolute error in per second (SAE/s) were adopted to quantitatively evaluate tracking performances, and the compared outcomes are presented in Table 2. By comparing the quantitative analysis results, the proposed control method presents a better control performance in the position and velocity tracking under the disturbance. In other words, the training served with the proposed control method is more stable and smooth, which is well indicated from the three evaluated 

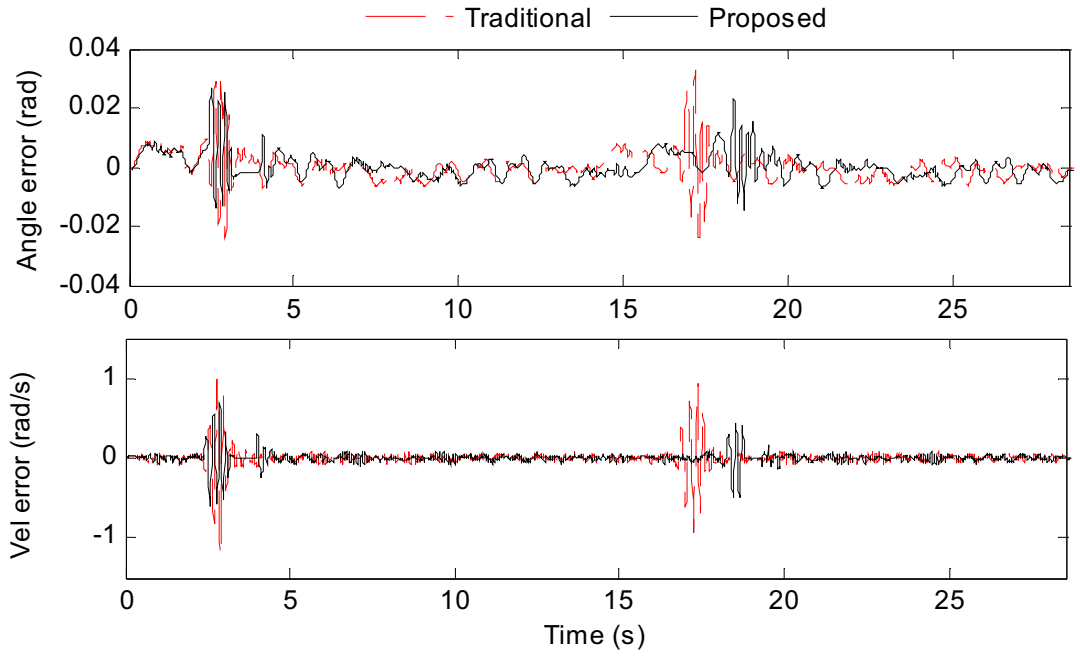

Fig. 5. Experiments with $10 \mathrm{~N}$ disturbance for a second.

Table 2

Tracking performance comparison under one-second $10 \mathrm{~N}$ disturbance

\begin{tabular}{llll|lll}
\hline & \multicolumn{3}{l|}{ Position error (rad) } & \multicolumn{3}{l}{ Vel error (rad/s) } \\
& MAE & SAE & SAE/s & MAE & SAE & SAE/s \\
\hline Proposed method & 0.0270 & 9.4489 & 0.3136 & 0.6947 & 108.8464 & 3.6126 \\
Traditional method & 0.0326 & 9.4867 & 0.3323 & 1.2318 & 131.7167 & 4.6135 \\
\hline
\end{tabular}

indices.

In the qualitative experiments, the subject was conducted to come into being different PSTIL (with some degree of disturbances) during the training, in order to verify the humanized dynamic regulation in the real-time motion speed and ROTM for the robot-assisted passive training. As introduced in section 3.2, the adjustment of the ROTM is based on the motion performances which are mainly reflected on the training speed, and the regulation of the real-time motion speed is a middle process. It means that the regulating of the speed is included in the adjusting process of the ROTM. Therefore, the experiments for adjusting ROTM are presented to verify the function of the humanized training. All the information was recoded to analyze the function of the humanized training, such as the control torque, the original and adjusted trajectories, the point numbers in the session, the regulation times for motion speed, the pausing times, the global speed coefficient, and the number of ideal training sessions. The experimental results of increasing and decreasing the ROTM are shown in Figure 6.

As shown in Figure 6, during the training, the control system is able to adaptively online adjust the ROTM or the global training speed. In Figure 6(a), the ROTM of the fourth session is adjusted up from 156 to 170 training points after two ideal training sessions, the second and third sessions. In Figure 6(b), there are many times of caused pausing in the first session, and then the ROTM is adjusted down for the next session from the original 203 training points to 136. Meanwhile, in Figure 6(b), the global predefined training speed coefficient of the fourth session is adjusted from 1.0 to 0.8 according to the regulating mechanism. The real-time training speed is well regulated according to the assessment results of the PSTIL, and the designed control system well manages the adjustment of the ROTM and global training speed, which is very consistent with the clinical therapy by physiotherapist. Motion training under suitable ROTM is helpful to improve the safety and the treatment efficacy. 

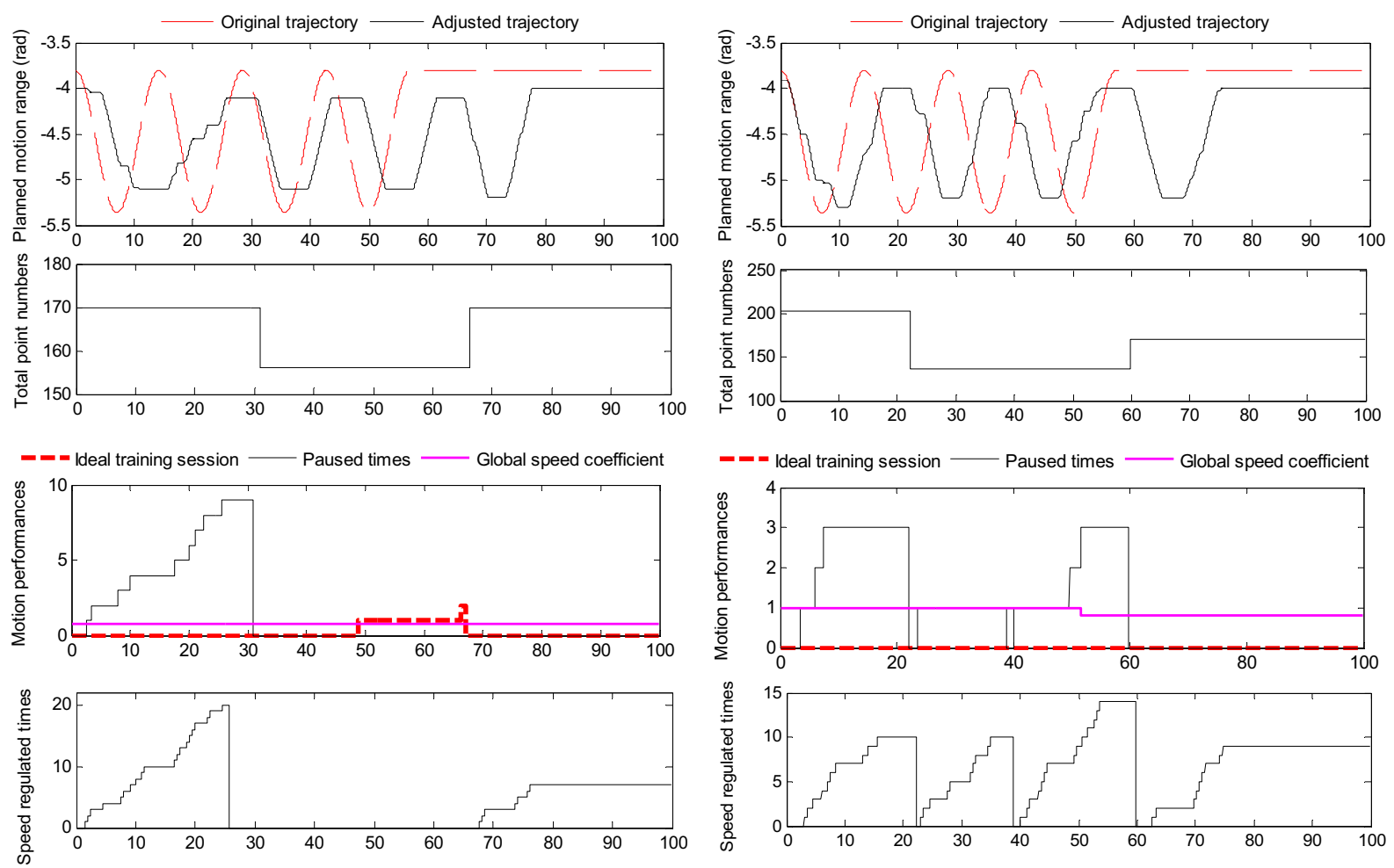

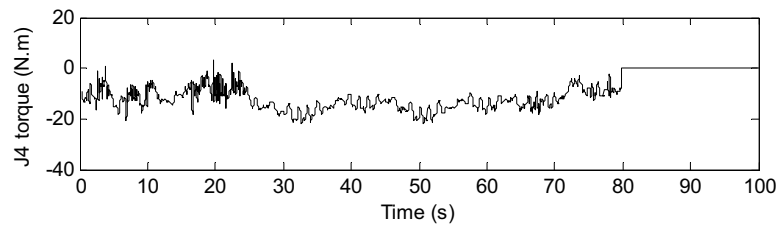

(a) Increasing the ROTM

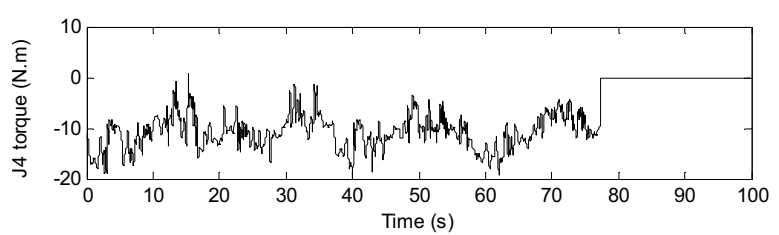

(b) Decreasing the ROTM

Fig. 6. Experiments for humanized trainings.

\section{Conclusion and discussion}

In this research, a novel control system design method combined with an online assessing-andadjusting section was proposed to achieve humanized passive training. During the training, based on the motion regulating mechanism, the real-time motion speed was regulated according to the assessment results of the PSTIL, and the ROTM was adjusted according to the motion performances. The quantitative and qualitative experiments were carried out to investigate the control performances in stability and humanization. The experimental results indicted that the designed rehabilitation system with the proposed method presented a better motion stability and smoothness than the traditional control method. Meanwhile, it was able to serve with an intelligent humanized passive training, just as what the physiotherapist did in clinical therapy, which was helpful to make the robot-assisted training more safety and efficacy. At current stage, this paper has investigated and verified the effectiveness for achieving the motion stability and humanization by the preliminary experiments with a healthy subject, not for validating the rehabilitation efficacy. Therefore, as a future work, we will begin pilot 
studies with stroke patients to further test the efficacy of the robot-assisted humanized passive training in clinical trials.

\section{Acknowledgment}

This work was supported by the National Natural Science Foundation of China (61325018), Jiangsu Ordinary University Science Research Project (14KJB510002) and the Foundation of Changzhou University (ZMF13020048).

\section{References}

[1] K. Daniel, C.D.A. Wolfe, M.A. Busch, et al., What are the social consequences of stroke for working-aged adults? A systematic review, Stroke 40 (2009), 431-440.

[2] K.J. Chisholm, K. Klumper, A. Mullins, et al., A task oriented haptic gait rehabilitation robot, Mechatronics 24 (2014), 1083-1091.

[3] Homepage of Chinese Ministry of Health: www.moh.gov.cn/public/, last accessed: Sep. $26^{\text {th }}, 2013$.

[4] A. Basteris, S.M. Nijenhuis, A.H.A. Stienen, et al., Training modalities in robot-mediated upper limb rehabilitation in stroke: A framework for classification based on a systematic review, Journal of Neuroengineering and Rehabilitation 11 (2014), 111.

[5] M.C. Laura and J.R. David, Review of control strategies for robotic movement training after neurologic injury, Journal of NeuroEngineering and Rehabilitation 6 (2009), 20.

[6] M. Casadio, I. Tamagnone, S. Summa, et al., Neuromotor recovery from stroke: Computational models at central, functional, and muscle synergy level, Computational Neuroscience 7 (2013), 1-14.

[7] M. Sivan, J. Gallagher, S. Makower, et al., Home-based Computer Assisted Arm Rehabilitation (hCAAR) robotic device for upper limb exercise after stroke: Results of a feasibility study in home setting, Journal of Neuroengineering and Rehabilitation 11 (2014), 163.

[8] N. Nordin, S.Q. Xie and W. Burkhard, Assessment of movement quality in robot-assisted upper limb rehabilitation after stroke: a review, Journal of Neuroengineering and Rehabilitation 11 (2014), 137.

[9] M.H. Rahman, M.J. Rahman, O.L. Cristobal, et al., Development of a whole arm wearable robotic exoskeleton for rehabilitation and to assist upper limb movements, Robotica 33 (2015), 19-39.

[10] M. Mihelj, Human arm kinematics for robot based rehabilitation, Robotica 24 (2006), 377-383.

[11] M.S. Ju, C.K. Lin, D.H. Lin, et al., A rehabilitation robot with force-position hybrid fuzzy controller: Hybrid fuzzy control of rehabilitation robot, IEEE Transactions on Neural Systems and Rehabilitation Engineering 13 (2005), 349-358.

[12] M.M. Zhang, T.C. Davies and S.N. Xie, Effectiveness of robot-assisted therapy on ankle rehabilitation - a systematic review, Journal of EuroEngineering and Rehabilitation 10 (2013), 30.

[13] M.K. O’Malley, A. Sledd, A. Gupta, et al., The rice wrist: A distal upper extremity rehabilitation robot for stroke therapy, Proceedings of International Mechanical Engineering Congress and Exposition, Chicago, IL, USA, 2006, pp. 1-10.

[14] P.R. Culmer, A.E. Jackson, S. Makower, et al., A control strategy for upper limb robotic rehabilitation with a dual robot system, IEEE/ASME Transactions on Mechatronies 15 (2010), 575-585.

[15] L.Z. Pan, A.G. Song, G.Z. Xu, et al., Hierarchical safety-supervisory strategy for robot-assisted rehabilitation exercise, Robotica 31 (2013), 757-766.

[16] A. Sawers and L.H. Ting, Perspectives on human-human sensorimotor interactions for the design of rehabilitation robots, Journal of EuroEngineering and Rehabilitation 11 (2014), 142.

[17] N. Hogan, Impedance Control: An approach to manipulation: Part 1-theory, Part 2-Implementation, and Part 3Application, ASME Journal of Dynamic Systems, Measurement and Control 107 (1985), 1-24.

[18] G.Z. Xu, A.G. Song and H.J. Li, Adaptive impedance control for upper-limb rehabilitation robot using evolutionary dynamic recurrent fuzzy neural network, Journal of Intelligent \& Robotic Systems 62 (2011), 501-525.

[19] M.M. Fateh and R. Babaghasabha, Impedance control of robots using voltage control strategy, Nonlinear Dynamics 74 (2013), 277-286.

[20] G.Z. Xu, A.G. Song and H.J. Li, Control system design for an upper-limb rehabilitation robot, Advanced Robotics 25 (2011), 229-251. 